\title{
Protocol
}

\section{Effect of a suite of aggression management modules on health professions student knowledge, skills and confidence: an investigator- blinded, parallel-group randomised controlled trial protocol}

\section{Steven Lau ${ }^{1 *}$, Katrina Recoche ${ }^{2}$, Robyn Fairhall ${ }^{2}$, Cameron Gosling ${ }^{3}$, Bernadette Hayman ${ }^{4}$, Primrose Lentin $^{5}$, Jim Snipe ${ }^{6}$, Shelley Turner ${ }^{7}$, Caroline Wright ${ }^{8}$, Allie Ford ${ }^{9}$, Kristin Lo ${ }^{1}$}

\author{
${ }^{1}$ Department of Physiotherapy, Monash University, Frankston Australia \\ ${ }^{2}$ Department of Nursing and Midwifery, Monash University, Frankston Australia \\ ${ }^{3}$ Department of Paramedicine, Monash University, Frankston Australia \\ ${ }^{4}$ Department of Occupational Health and Safety, Monash University, Frankston Australia \\ ${ }^{5}$ Department of Occupational Therapy, Monash University, Frankston Australia \\ ${ }^{6}$ Five arcs, Australia \\ ${ }^{7}$ Department of Social Work, Monash University, Caulfield Australia \\ ${ }^{8}$ Department of Medical Imaging, Radiation Therapy and Radiation Sciences, Monash University, Frankston Australia \\ ${ }^{9}$ Education Academy, Monash University, Frankston Australia
}

Received: 28 July 2021

Revised: 10 September 2021

Accepted: 12 October 2021

\section{*Correspondence:}

Mr. Steven Lau,

E-mail: steve.lau@monash.edu

Copyright: (C) the author(s), publisher and licensee Medip Academy. This is an open-access article distributed under the terms of the Creative Commons Attribution Non-Commercial License, which permits unrestricted non-commercial use, distribution, and reproduction in any medium, provided the original work is properly cited.

\section{ABSTRACT}

Background: Incidents of aggression are increasing in healthcare. Students are at higher risk of being on the receiving end of client aggression due to their lack of experience. Students also do not feel prepared to manage these situations.

Methods: Students of health professions in the School of Primary and Allied Health Care will be eligible and contacted within lectures/via the learning management system Moodle. A randomised controlled trial (RCT) will be conducted to evaluate a suite of online aggression management modules for health professions students prior to entering their clinical years of study. If students are able to undertake face to face simulations they will be assessed by academic staff members using the English modified de-escalating aggressive behaviour scale (EMDABS). Mock clients will assess the students using the consultation and relational empathy (CARE) measure. Students will complete a self-assessment using the confidence with coping with patient aggression instrument and a knowledge questionnaire.

Conlusions: This RCT will provide novel information on the effectiveness of delivering a suite of online aggression management modules to health professions students. There are three separate modules each of approximately 30 minutes duration such that the attention of students is maintained. The three modules are: 1. Recognising and preventing aggression-the basics, 2. Responding to aggression-the basics, 3. Aggression in healthcare-the basics (Case study).

Trial registration number: This trial has been registered with the Australian and New Zealand clinical trials registry (ANZCTR) ACTRN12621000382875.

Keywords: Aggression, Health professions student, Clinical education, Risk management 


\section{INTRODUCTION}

A behaviour that is increasing in health care is aggression against health care providers. ${ }^{1,2}$ The potential ramifications are serious including both physical and mental injury and even death. ${ }^{3}$

\section{Existing knowledge}

Younger health professions students with less experience are at a higher risk of being on the receiving end of client aggression due to their inexperience. ${ }^{4}$ Students do not feel confident to manage situations involving client aggression. $^{5}$ It is therefore imperative that we teach our health professions students about managing aggressive clients.

\section{Need for trial}

In a 2020 systematic review on aggression management education in health professions students there were no randomised controlled trials found involving aggression management strategies for these types of students. ${ }^{6} \mathrm{We}$ aim to fill this gap by running a randomised controlled trial evaluating a suite of online aggression management modules for health professions students early in their education.

The primary research question: What is the influence of a suite of basic aggression management modules on beginner health professions student knowledge, skills and confidence?

The hypothesis is that students' knowledge, skills and confidence to manage a situation involving an aggressive client will improve after undertaking the modules.

\section{METHODS}

This protocol was informed by the CONSORT 2010 checklist of information to include when reporting a randomised controlled trial. ${ }^{7}$ It was also informed by the standard protocol items: recommendations for interventional trials (SPIRIT) documents and checklist. ${ }^{8}$

Due to the COVID-19 panademic we have needed to have a plan A and plan B for this study as our funding was revoked. Plan A involves a face to face study \pm funding. Plan B involves an off campus study \pm funding.

\section{Pilot}

We will pilot the module with up to 30 student volunteers each from the masters of radiation therapy and masters of social work. This will involve completing the suite of modules and attending a focus group of up to 60 minutes. The focus group/s will be recorded, transcribed and anonymised prior to content analysis that will be used to create themes regarding exploration of student experiences of completing the module. ${ }^{9}$ This will help us improve the module before it is used in the RCT. For the content analysis, two researchers will independently theme the qualitative comments and in colaboration discuss the theming framework. After deciding on a final list of themes the researchers will reach concensus on these themes and decide on illustrative quotes.

The focus group questions are: Can you share with me how you found navigation through the modules? Can you please comment on the pace of the modules/browsing speed and how long it took to work through the content? What are your thoughts about how the module was structured? What aspects did you like in terms of the structure and what would you change? Can you share your thoughts on the content that was delivered in the module, in terms of relevance, how engaging it was, how clear and easy to read it was? What key learning did you take from the module, which aspects do you remember when you reflect on the content? When and how might the learning help you on your clinical placement? Any suggestions on how to improve the module?

\section{Recruitment}

Students will be recruited in lectures or via the learning management system (Moodle) by members of the research team. Students will review the explanatory statement before deciding whether to complete the initial survey (Figure 1).

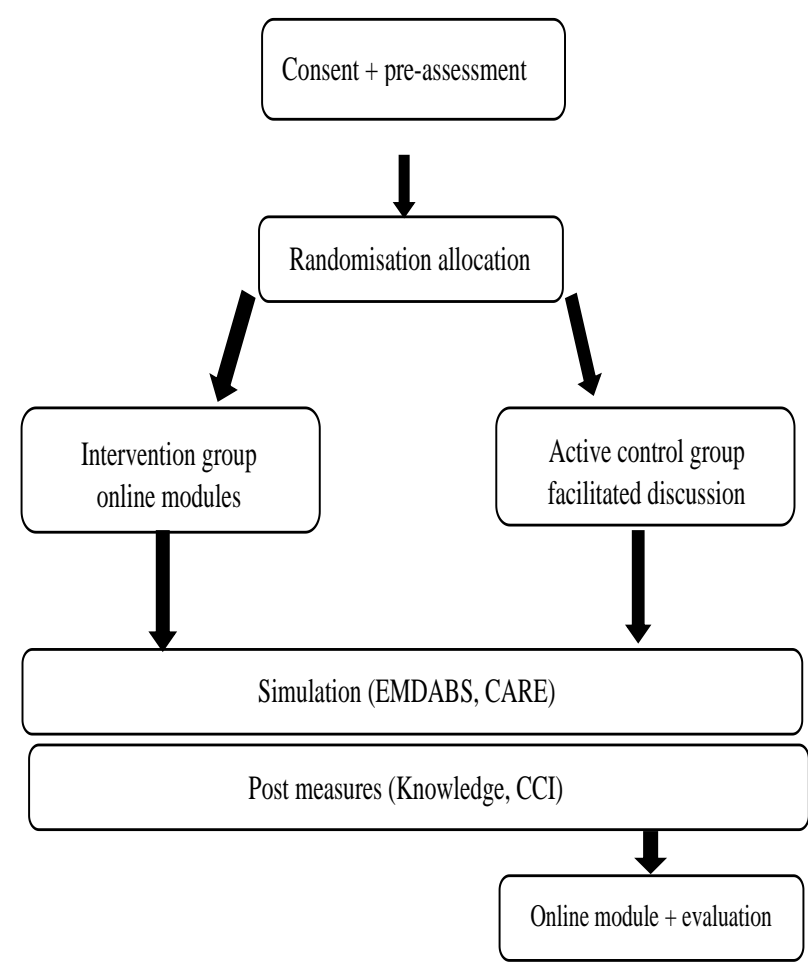

Figure 1: Flow diagram of proposed randomised controlled trial plan A, for plan B omit the simulation or provide an online sim. 


\section{Trial}

The trial design will be a randomised controlled trial with: Study group: $50 \%$ of participants will complete a suite of online aggression management modules consisting of three parts of approximately 30 minutes duration each and control group: $50 \%$ of participants will be allocated to the active control intervention (a 90 minute facilitated discussion about situations in which the students have experienced aggression themselves, seen or been on the receiving end of acts of the aggression).

\section{Participants}

\section{Inclusion criteria}

Students in all allied health professions degrees of the school of primary and allied health care at Monash University will be recruited by presentations in lectures and/or contact via the learning management system Moodle. Students with additional training in aggression (eg: students who have been in security positions, previous clinical positions, positions in retail that have had additional training in coping with aggressive people, any other education on aggression management) will be identified either before the commencement of the RCT or after completion of the pre-survey. This will be taken into account when analysing the data.

\section{Exclusion criteria}

Students who have already participated in any clinical experience at the university will be excluded from the trial.

\section{Settings and location where data collected}

Data will be collected at Monash University, Frankston, Victoria, Australia in a simulation lab or online via zoom if required.

\section{Randomisation}

If interested in the study, students will complete a preassessment containing demographic information and the pre-intervention assessment tools eg: knowledge assessment and confidence measures. They will then be allocated by a random number generator to the intervention or active control groups. This will be completed using Microsoft Excel ${ }^{\mathrm{TM}}$ and concealed by a research assistant such that the research team will be unaware of the allocation of participants. We intend to have a similar ratio of genders in each group given that there may be gender differences in communication skills. ${ }^{10}$ The research assistant will assign the interventions to participants who will be blinded to the intervention as much as is practically possible.

\section{Intervention}

The intervention is a suite of online aggression management modules (three modules of approximately 30 minutes duration each) which have been constructed using articulate RISE embedded into our learning management system Moodle. The modules were developed by the research team with assistance of an educational designer (AF). The active control is a 90 minute discussion about participants' experiences with aggression whether feeling aggressive themselves or witnessing or being on the receiving end of acts of aggression. The discussion will be facilitated by a member of the research team with questions such as "Describe when you have felt aggressive, describe a situation that you felt was aggressive, what was it that made you feel that aggression was being demonstrated, what's your definition of aggression, what did you do in this situation, with hindsight what might you have done differently?

Plan A: The students will then all be assessed using a simulation of an aggressive client. The simulation duration will be up to 10 minutes plus 5 minutes self assessment time. The simulation will involve a mock patient with pain played by a health professional or actor. The client will be frustrated by the waiting time and the fact that they are being seen by a student. Given this study will be completed by health professions students of a number of disciplines they will each do a subjective assessment only according to how they have been taught in each of their own professions. No objective assessments of the client will be done as these are diverse across the health professions. The simulation will be assessed by members of the research team who will be blinded to group allocation. Students in the control group will be given access to the online module after their postassessment evaluations have been completed.

Plan B: Students will be assessed online with or without simulation.

\section{Outcome measures}

The demographics will be collected in a pre-survey via qualtrics the link will be provided on Moodle. We will record student age, gender, health professions discipline, current year of the course, whether they have had any clinical experience in university and type (this will be used to include or exclude participants), whether they have had any experience in customer service/security or a job that has experience in dealing with people who are aggressive and type of experience they have had, previous code grey or aggression training, previous experience being on the receiving end of an act/s of aggression.

Knowledge: Student knowledge will be tested with a multiple choice quiz of approximately 5-10 min duration that has been developed by the research team as no 
validated aggression management knowledge tests have been published.

Skills: Plan A-Students will be assessed by the academic staff members using the de-escalating aggressive behaviour scale (DABS). ${ }^{11,12}$ The EMDABS is an English version adapted from the German which has 7-items: valuing the client, reducing fear, enquiring about the client's fears and anxieties, providing guidance to the client, working out possible agreements, remaining calm, securing one's own safety. It uses a 5-point Likert scale ranging from strongly disagree to strongly agree with descriptive anchors for 1, 3 and 5. The last item, number 7 is reverse scored. Overall higher scores indicate increased ability to de-escalate aggression. Literature demonstrates Cronbach's alpha 0.901 which indicates very good internal consistency. ${ }^{13}$ It has been previously used to evaluate responses to aggression management modules completed by nursing students. ${ }^{12}$ The EMDABS has also demonstrated very good inter-rater reliability $(\operatorname{ICC}(3,1)=0.752) .{ }^{14}$ Before assessing the simulation, the assessors who will be using the tool will assess a video of a student interaction and score this and then discuss their scores to come to a consensus. This aims to improve inter-rater reliability.

Mock clients will assess the students using the consultation and relational Empathy (CARE) measure. ${ }^{15}$ This tool has 10 items. Each score describes how the health professional was at, for example, making the client feel at ease. This is rated on a 5-item Likert scale with poor $=1$, fair $=2$, good $=3$, very good $=4$, excellent $=5$. The internal reliability of the CARE was high (Cronbach's alpha 0.92). ${ }^{15}$ It also correlates with other measures of empathy. The mock clients will be able to mark a video of a student performing a subjective prior to the simulations to improve interrater reliability.

Plan $B$-The EMDABS and CARE team will only be assessed if a simulation is completed otherwise students will have only their knowledge and perceptions assessed.

Confidence: Plan A and B: At the end of the study students will complete a self-assessment using the confidence with coping with patient aggression instrument $(\mathrm{CCI}) .{ }^{16}$ This has ten items eg: "How comfortable are you working with an aggressive patient?". It uses an 11-item Likert scale with anchors eg: $1=$ very uncomfortable, $11=$ very comfortable. Higher scores=more confidence in coping with patient aggression. It has demonstrated the ability to monitor short and long-term change by Thackrey. The Cronbach's alpha was found to be 0.92 . $^{16}$

In order to evaluate the quality of the online module, we will utilise questions from the publication by Zhao et $\mathrm{al}^{17}$ : I was satisfied with the delivery medium more specifically ease of the navigation and movement around the site. I was satisfied with the quality of the module. Any suggestions on how to improve the module? And
Any suggestions on how to improve this satisfaction survey?

Questions from the publication by Chua and Lam will also be used which measures the responses to the following questions using a 5-point Likert scale from $1=$ strongly disagree to $5=$ strongly agree: ${ }^{18}$ The module was structured in a way that helped me to understand. The content of the subject was relevant to my learning needs. The content of the subject was engaging, the writing style of the subject material was clear and easy to read. Using a 5-point Likert scale (1=poor, 5=excellent): Overall, how would you rate the quality of your learning from this module? Overall, how would you rate your satisfaction level with this module?

We will also add some further questions such as: How long did it take you to do the module? What time in your course do you think this module would best fit? Eg: semester 1 year 1 etc. This will help us decide on the best timing of the education. What was the take home message from this module? This will give us insight into what the students are understanding from the modules. How many parts of the module did you complete fully? This will tell us whether the students were not interested / engaged and just completed one section of the module. How long did it take to complete each section of the module? This is to help us to more accurately inform future students of the time necessary to put aside to complete the modules and anything else you would like to add about the module?

\section{Post RCT}

Students in the active control group will be given access to the online module after the RCT is complete so that they are not disadvantaged. Both groups will do a survey to evaluate the content/quality of the online modules after completing them.

\section{Plans to promote retention}

We will send reminders to students via the learning management system Moodle to promote completion of study requirements. Students who discontinue will have their pre-data deleted.

\section{Data management}

Details of data management procedures can be found in the explanatory statement. These include keeping data in a secure file to be stored in lab archives for 5 years and be shared only with the research team. The data will be made anonymous by the research assistant who will know the group allocation and identification of the participants. The research team, which has a representative from each health professions discipline from within the School of Primary and Allied Health care, will have access to the deidentified data. The excel file will be deleted after 5 years. 


\section{Missing data}

Where possible we will delete the data of students who did not complete the study. We will use intent to treat analysis.

\section{Sample size}

The sample size required was calculated using G-power to be a total of 138 students per group. The power calculation to determine sample size was based on the confidence in coping with patient aggression instrument data as published by Grenyer et al. ${ }^{19}$ The data that was used was pre-training mean 69.85, standard deviation 13.99 , post training mean 82.15 , standard deviation 9.2, $\mathrm{t}=4.38, \mathrm{p}=0.00$, alpha $=0.05$, power (beta) $=0.80$. We used a matched pairs design.

\section{Analyses}

Data will be collated and assessed for normality and skewness. Data will be reported using frequency, mean (standard deviation), and medians (interquartile range) where appropriate. The difference in the results of the pre and post outcome measures will be analysed using parametric or non-parametric statistics as appropriate. Analysis will be performed using an intention to treat approach. Further sub group analysis will be included adjusting for gender, year level and current allied health course enrolment. Items that are identified as significant in univariate testing will be entered into a multivariate ordinal logistic regression model. The strength of association will be reported using odds ratios (OR) and 95\% confidence intervals (CIs). All tests will be twotailed unless otherwise stated, and results will be considered statistically significant if the $\mathrm{p}$ value is less than 0.05 .

\section{Data display}

A Table will be included showing the baseline demographic and clinical characteristics for each group. Means and standard deviations will be displayed.

\section{Sharing of outcomes}

Results may be shared in journals, conferences, reports to participants, book chapters or part of a thesis. Results will be made available upon request to participants in a totally deidentified summary form, in which no individual can be identified.

\section{Harms/unintended effects}

We will comment on whether there have been any harms or unintended effects reported for any of the students. Contact details of the university counsellors are included on the explanatory statement.

\section{Auditing}

Data collection will be monitored by a research assistant.

\section{Registration}

The trial has been registered with the Australian and New Zealand clinical trials registry (Registration number: ACTRN12621000382875).

\section{Funding}

No funding was required for this study.

\section{Incentives}

If funds are available we will put participants into a draw to receive one of $20 \$ 50$ gift vouchers as an incentive to complete the study. This is to say thank you for their time and participation. This is a minimal amount and will not result in people putting themselves at risk to obtain financial gain.

\section{Ethical approval}

Ethical approval for this study was granted by the Monash University Human Research Ethics Committee in October 2020 with reference number 23695.

Any protocol modifications which may make an impact on the conduct of the study including changes of study objectives, design, patient population, sample sizes, study procedures, or significant administrative aspects will require a formal amendment to the protocol and approval by the relevant ethics committee. Such amendments will be agreed upon by research team prior to implementation.

\section{Consent or assent}

Only students who consent to the research will be included. This is implied consent- completing the surveys will imply that students have given consent.

\section{Confidentiality}

Personal information about the participants will be collected using survey creation software (Qualtrics), the research assistant will deidentify data to protect the confidentiality of the participants. We will also use a code word identifier that the student devises to connect pre and post data from each individual. The code word identifier will be the first 3 letters of Mum's maiden name and first 3 letters of their street name.

\section{Declaration of interests}

The research team has declared that they have no conflicts of interest. 


\section{Ancillary and post-trial care}

All students will have access to the suite of aggression management modules at the end of the trial.

\section{Trial results}

The trial results will be published in a medical education journal and may be presented at a relevant conference.

\section{Authorship}

The authors of any subsequent publication will be decided by the research group prior to submission based on their contributions to the manuscript.

\section{Reproducible research}

The data will not be made available to others in accordance with our ethics approval.

\section{DISCUSSION}

The primary purpose of this RCT is to evaluate a suite of online aggression management modules for health professions students. This RCT will also provide valuable information with regard to student self-perceived confidence and how this impacts on their actual skill competency as assessed with a simulation. Our research team suggested that experience in customer service or security roles and maturity of age may influence a student's competence in aggression management skills due to having more life experience so we will obtain data on these factors to enable sub-analysis. This RCT has the potential to further explore education regarding aggression management which may assist in further refining curriculum within health professions courses.

\section{ACKNOWLEDGEMENTS}

The authors would like to acknowledge the Traditional Custodians of the land on which we live and work and pay our respects to Elders, past, present and future.

Funding: No funding sources

Conflict of interest: None declared

Ethical approval: The study was approved by the Institutional Ethics Committee

\section{REFERENCES}

1. Morphet J, Griffiths D, Beattie J, Reyes DV, Innes $\mathrm{K}$. Prevention and management of occupational violence and aggression in healthcare: a scoping review. Collegian. 2018;25(6):621-32.

2. Schablon A, Wendeler D, Kozak A, Nienhaus A, Steinke S. Prevalence and consequences of aggression and violence towards nursing and care staff in Germany-A survey. Int $\mathrm{j}$ environmental res public health. 2018;15(6):1274.

3. Morrison JL, Lantos JD, Levinson W. Aggression and violence directed toward physicians. J general internal med. 1998;13(8):556.

4. Baxter E, Hafner RJ, Holme G. Assaults by patients: the experience and attitudes of psychiatric hospital nurses. Aus N Zea J Psychiatry. 1992;26(4):567-73.

5. Lo K, Storr M. Physiotherapy Students' Exposure to Confronting Clinical Situations-A Qualitative Review. J Allied Health. 2020;49(4):153E-60E.

6. Lau S, Nicholls R, Tse Y. Effect of curriculum on students' management of aggression: a metaanalysis. 2020;2021.

7. Schulz KF, Altman DG, Moher D, Group C. CONSORT 2010 statement: updated guidelines for reporting parallel group randomised trials. Trials. 2010;11(1):32.

8. Chan A-W, Tetzlaff JM, Altman DG. SPIRIT 2013 statement: defining standard protocol items for clinical trials. Ann internal med. 2013;158(3):200-7.

9. Braun V, Clarke V. Using thematic analysis in psychology. Qualitative res psychol. 2006;3(2):77101.

10. van den Brink-Muinen A. The role of gender in healthcare communication. Patient Education Counseling. 2002;48(3):199-200.

11. Nau J, Halfens R, Needham I, Dassen T. The deescalating aggressive behaviour scale: development and psychometric testing. J Adv Nursing. 2009;65(9):1956-64.

12. Nau J, Halfens R, Needham I, Dassen T. Student nurses' de-escalation of patient aggression: A pretest-posttest intervention study. Int $\mathrm{j}$ nursing studies. 2010;47(6):699-708.

13. Nau J, Walter G, Oud N. Measuring de-escalation by using the De- escalating Aggressive Behaviour Scale (DABS). J Adv Nurs. 2019:65(9)263-6.

14. Mavandadi V, Bieling P, Madsen V. Effective ingredients of verbal de- escalation: validating an English modified version of the 'De- Escalating Aggressive Behaviour Scale'. J psychiatric mental health nursing. 2016;23(6-7):357-68.

15. Mercer SW, Maxwell M, Heaney D, Watt G. The consultation and relational empathy (CARE) measure: development and preliminary validation and reliability of an empathy-based consultation process measure. Family practice. 2004;21(6):699705.

16. Thackrey M. Clinician confidence in coping with patient aggression: Assessment and enhancement. Professional Psychol Res Practice. 1987;18(1):57.

17. Zhao F. Enhancing the quality of online higher education through measurement. Quality assurance in Education. 2003;26(1):84-6.

18. Chua A, Lam W. Quality assurance in online education: The Universitas 21 Global approach. Bri J Educational Technol. 2007;38(1):133-52. 
19. Grenyer BF, Grenyer BF, Ilkiw-Lavalle O. Safer at work: development and evaluation of an aggression and violence minimization program. Aus $\mathrm{N}$ Zea J Psychiatry. 2004;38(10):804-10.
Cite this article as: Lau S, Recoche $\mathrm{K}$, Fairhall R, Gosling C, Hayman B, Lentin P et al. Effect of a suite of aggression management modules on health professions student knowledge, skills and confidence: an investigator -blinded, parallel-group randomised controlled trial protocol. Int J Clin Trials 2021;8(4):308-14. 